\title{
Results of the Multimessenger GRB Observations in the Lomonosov Mission
}

\section{Vitaly Bogomolov ${ }^{1}$, for Lomonosov-GRB collaboration}

Lomonosov Moscow State University, Skobeltsyn Institute of Nuclear Physics, Physical department 1(2), Leninskie gory, GSP-1, Moscow, Russia

E-mail: bogoviterambler.ru

\begin{abstract}
GRB study is one of the main goals of the Lomonosov space mission. Multimessenger GRB observation is the only way of progress achievement in their study. The Lomonosov satellite is the first space mission in which the multi-wave length observations of GRBs are realized in real time without necessity of optical instrument re-orientation on GRB monitor trigger. The mission payload includes the GRB monitor BDRG, wide - field optical cameras SHOK and UFFO instrument consisting of X-ray imaging telescope UBAT and UV slewing mirror telescope SMT. Two wide field cameras are placed in such a way that their fields of view (FOV) are overlapped by the GRB monitor detector FOVs. This allows the simultaneous GRB observations in gammas and optics in all-time scale of event evolution including obtaining optical light curves of prompt emission as well as of precursors. The real time data transfer to the GCN for detected GRB is realized as well as operative control of BDRG data on triggers from ground based facilities including neutrino and gravitation wave detectors. The GRB catalogue is presented in this report, also those events, for which information was obtained in different bands, are discussed in details.
\end{abstract}




\section{Introduction}

Multi-wavelength GRB observation is one of the main goals of the Lomonosov space mission launched on 28.04.2016 to the solar synchronous orbit. The mission payload includes a complex of instruments developed for GRB prompt emission detection in all kinds of radiation [1][2]. It consists of gamma-ray monitor BDRG, wide field optical cameras SHOK and UFFO instrument consisting of X-ray imaging telescope UBAT and UV slewing mirror telescope SMT. In all of the experiments before Lomonosov mission the pointing of optical and soft $\mathrm{x}$-ray instruments after the trigger produced by gamma-telescope is used for multi-wavelength GRB observations. Several methods are used to provide the observation on early phase of GRB source activity. Fast transfer of the message with GRB coordinates to the ground telescopes on the trigger, produced by space instruments was realized by Fermi/GBM, Swift/BAT and some other experiments [3]. Fast pointing of all instruments after GRB trigger by the rotation of the satellite was realized in Swift mission. The time of the satellite reorientation about several tens of seconds allowed one to study GRB afterglows on early stages [3], but the beginning phase of the GRB could not be observed by this method. The Lomonosov satellite is the first space mission in which the early optical observations of GRBs are realized without optical instrument reorientation on GRB monitor trigger by real time recording of the sequence of images from wide-FOV cameras. The real time GRB data transfer to the ground via GlobalStar modem is also realized in Lomonosov mission. Joint analysis of the satellite data and the data of ground robotic telescopes is very useful as well as the operative control of BDRG gamma-ray data on triggers from ground based facilities including neutrino, UHECR and gravitation wave detectors.

\section{Instruments onboard Lomonosov Satellite}

The gamma-ray spectrometer named BDRG consisting of 3 orthogonaly directed detector units and two wide field cameras named SHOK are placed in such a way that their fields of view (FOV) are overlapped by the GRB monitor detector FOVs (see fig.1, left). The optical data can be stored by the trigger from gamma-detector. This allows multi-wavelength GRB observations in all-time scale of event evolution including optical light curves of prompt emission as well as of precursors.

The third GRB instrument onboard Lomonosov satellite named UFFO is designed as a combination of a coded-mask hard x-ray telescope UBAT and an optical telescope SMT with moving mirror that can be pointed to the GRB source for $\sim 1 \mathrm{~s}$. Its characteristics, operation modes and first results will be discussed in separate paper.

\subsection{BDRG gamma-ray monitor}

The BDRG gamma-ray spectrometer [4] is designed to obtain the temporal and spectral information of GRBs in the energy range of $10-3000 \mathrm{keV}$ as well as to provide GRB triggers on several time scales (10ms, $1 \mathrm{~s}$ and 20s) for ground and space telescopes, including the UFFO and SHOK. Three identical detector boxes of BDRG are oriented such a way that their axes are shifted by $90^{\circ}$ from each other. The comparison of their readings allows one to localize a GRB source in the sky with an accuracy of $\sim 2^{\circ}$ for bright GRBs. 

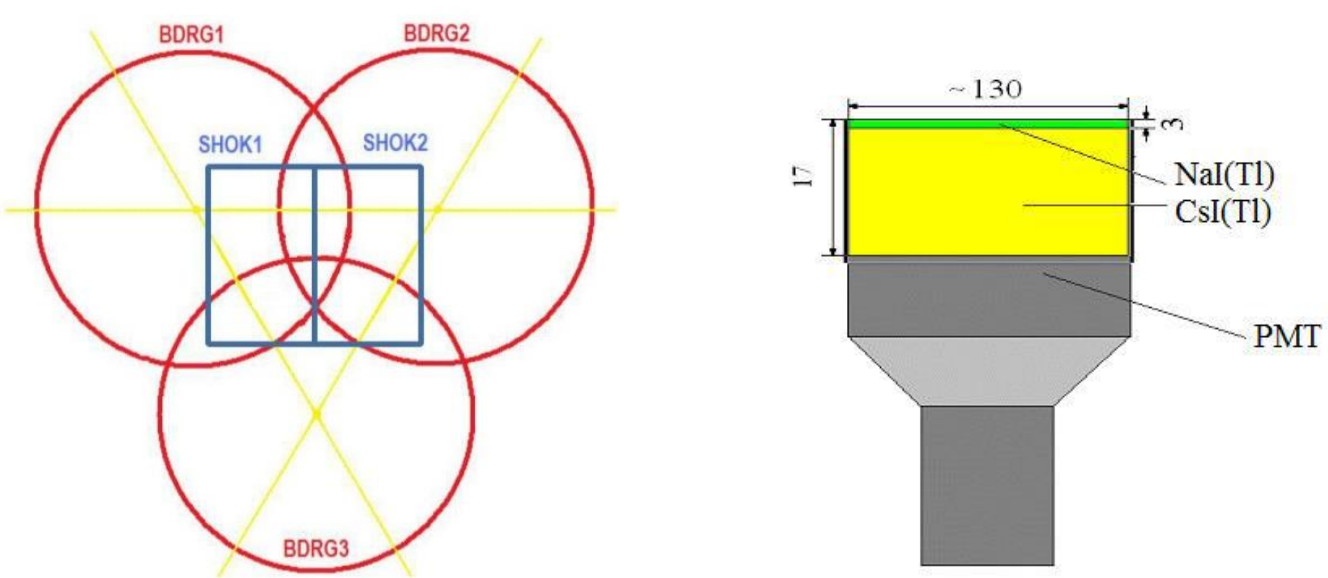

Fig.1. Left: the mutual position of BDRG detector units and SHOK wide field optical cameras FOV projections. Right: the design of BDRG scintillation detector

Each BDRG box is based on a phoswich $\mathrm{NaI}(\mathrm{Tl}) / \mathrm{CsI}(\mathrm{Tl})$ scintillator detector (see fig.1, right). A CsI(Tl) crystal in size of $\varnothing 130 \times 17 \mathrm{~mm}$ is placed underneath the thin $3 \mathrm{~mm} \mathrm{NaI(Tl)}$ as an active shield in the soft energy range and as the main detector in the hard energy range. The ratio of the $\mathrm{CsI}(\mathrm{Tl})$ to $\mathrm{NaI}(\mathrm{Tl})$ event rates at varying energies can be employed as an independent metric to distinguish GRB detections from the imitations originating from electrons in near-Earth vicinities.

The data from three detectors are collected in a BA BDRG information unit, which generates a GRB trigger and a set of data frames in output format. The scientific data output is $\sim 500 \mathrm{Mb}$ per day, including $\sim 180 \mathrm{Mb}$ of continuous data and number of $\sim 5 \mathrm{Mb}$ sets of detailed information for each burst-trigger. Continuous data contain count rates in 16 channels for each detector with $100 \mathrm{~ms}$ time resolution as well as detailed energy spectra. Trigger data contain a large portion of information recorded in gamma by gamma mode up to 1 million events for a trigger.

\subsection{SHOK optical cameras}

Lomonosov satellite is equipped with two identical devices SHOK 1 and SHOK 2 [4]. Each unit is based on a recording camera with an optical lens Nikkor attached to it. The camera linear dimensions are $110 \times 66 \times 66 \mathrm{~mm}$, lens $-\varnothing 69 \times 48 \mathrm{~mm}$. It is equipped with a CCD matrix Kodak KAI-11002 TrueSense with a resolution of $4008 \times 2672$ pixels at a pixel size of 9 microns. The camera has maximum quantum efficiency of $50 \%$ at a wavelength of $500 \mathrm{~nm}$. The camera provide a field of view of 1000 square degrees. At the same time the average quality of the image (FWHM - width at half intensity) along the field of view is FWHM _ 3 pix.

Each SHOK unit is a fast-acting wide-angle camera, which provide recording of optical emission with maximum magnitude of $9-10 \mathrm{~m}$ at a single frame and exposure time of 0.2 seconds. While operation cameras are continuously shooting the "film", the part of which is to be transmitted to the Earth at the gamma-ray burst detection. Taking into account very large amount of optical data two modes of data selection were realized. First one is the trigger mode. In this case several tens of seconds of the whole FOV "film" before and after the trigger moment are stored and then transmitted to the Earth. The trigger mode is activated after any 
alert-level BDRG trigger independently on estimated GRB coordinates. The probability that the source will be located in the cameras FOV is not high and the expected rate of such events is about once per 1-2 months.

The second mode is the continuous one. The image processing by powerfull computer inside of the SHOK unit is performed for optical transients search by real time differential frame processing. As a result, the difference frames containing strongly brightening objects and all moving objects (nearest asteroids, satellites, space debris) are stored. The information about all new opened objects and the images of the brightest of them is sent to Earth for further analysis during the day. Cameras are fixed to the spacecraft platform and rotate therewith. Thus, the whole sky is scanned.

\section{GRB observations in Lomonosov mission}

Lomonosov satellite was launched on $\sim 500 \mathrm{~km}$ polar orbit so gamma-ray detectors readings demonstrate the increases of the background when the satellite passes inner and outer radiation belts. Gamma-ray bursts can be detected in equatorial and in polar regiones where the background is low. However it is necessary to note that the burst-like events in the detector caused by the secondary gammas produced by precipitated electrons can appear in polar zones. In order to achieve better GRB sensitivity the triggering was realized at two levels. The threshold of the first level was chosen considerably low but only detailed gamma-ray data were stored by such trigger. About several tens of triggers of that type occured per day. Most of them were caused by particles. Second level - so called alert level - triggering has much higher threshold and some additional conditions such as the demand of suitable geografic coordinates of the piont of trigger production. When the alert trigger occur the data of SHOK cameras are fixed and a fast telegram is sent to the Earth via GlobalStar modem.

Two methods of GRB search were used. First one is the detailed analysis of the data stored by BDRG trigger. After excluding the imitations produced by particles the short list of candidates was compared with GCN notes as well as with GOES data on Solar activity. A number of GRBs was confirmed and several outburst of SGR 1935+2154 were also observed. All BDRG triggers concerning astrophysical GRBs were connected with the event also observed by some space instrument of the world scientific society. Another method was to search for the response in the monitoring channels at the moments of the GRBs listed in GCN. Several nontrigger events were added to the Lomonosov GRB catalog. Most of them were present at first months of the experiment during the period of the flight test and optimization of BDRG trigger algoritm.

There are $19 \mathrm{GRBs}$ in the Lomonosov/BDRG catalog for the period of operation from June 2016 to January 2017. All of them are long/soft gamma-ray bursts. The diagrams in fig.2 present the statistics of detected GRBs and the observational conditions. The estimated probability to detect a GRB listed in GCN is $~ 20 \%$ taking into account shadowing by the Earth and excluding the radiation belt regions. The probability to detect a GRB not listed in GCN is less than $10 \%$ because there is a lot of space missions such as Swift, Fermi, Wind-Konus and other continuously observing all the sky. However there are some GRBs in Lomonosov/BDRG catalogue that were not observed by the main specialized missions, for example GRB 160908A was observed only in CALET experiment [5]. 


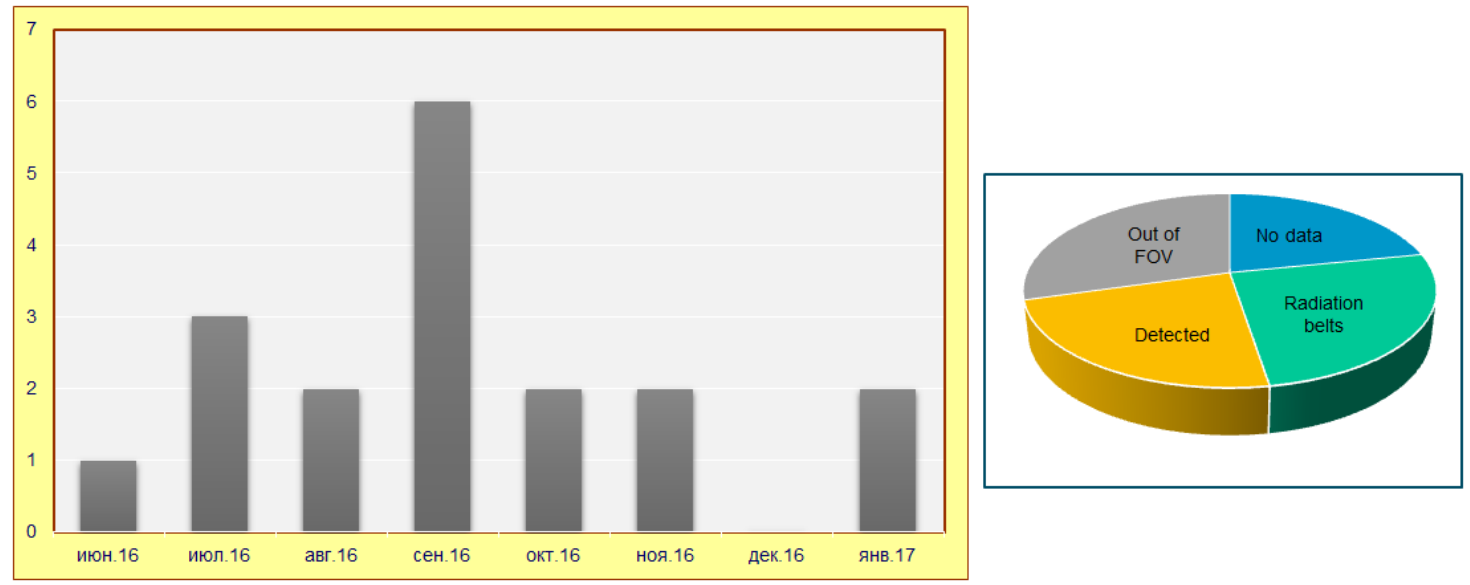

Fig. 2. Left: Month amount of GRBs observed by BDRG/Lomonosov from June, 2016 to Jan, 2017. Right: Statistics of observational conditions in Lomonosov/BDRG experiment for GRBs that occurred from June, 2016 to Jan, 2017

Total list of 19 GRBs observed in Lomonosov experiment is published on the oficial site of the mission [6]. No one of the sources of the bursts appeared in SHOK cameras FOV when this optical instrument was operating. However three times the bursts detected by Lomonosov/BDRG were observed by ground robotic telescopes of the net MASTER, developed by scientists of Moscow State University. In two of them no optical transient was discovered. Correspondent upper limits were obtained: 16.5 st.mag for GRB 160720A at 30794s after trigger [7] (the weather conditions were not fine in this case) and 18 st.mag for GRB 160824B at $1165 \mathrm{~s}$ after trigger [8]. In the case of GRB 161017A an optical transient was discovered. The result of this observation is presented in details in the next section.

\section{Multimessanger Observations of GRB 161017A}

The powerfull gamma-ray burst was detected by BDRG/Lomonosov on October 17, 2016. The burst was detected at significant levels $4.4 \sigma, 14.1 \sigma, 0.045 \sigma$ in $20-100 \mathrm{keV}$ range of $\mathrm{NaI}(\mathrm{Tl})$ detectors of BDRG-1, BDRG-2 and BDRG-3 units respectively, Thus, it was detected significantly by two detectors (BDRG-1 \& BDRG-2) with most significant response in BDRG-2 outputs. Detailed gamma by gamma data were recorded in the $30 \mathrm{~s}$ time interval before the trigger and in the $119 \mathrm{~s}$ time interval after.

The Light curve of GRB 161017A in the 20-300 keV range is presented at fig. 3. This curve is obtained from BDRG-2 $\mathrm{NaI}(\mathrm{Tl})$ outputs with $1 \mathrm{~s}$ time resolution. One can see that it is a long GRB with complex light curve consisting of several periods of gammaradiation. One can see at least 3 periods of GRB activity at $-30 \mathrm{~s} . . .-20 \mathrm{~s},-10 \mathrm{~s} . . .0 \mathrm{~s}$ and $100 \mathrm{~s} . .110 \mathrm{~s}$. The presence of gamma-ray flux in the last mentioned period indicates that the central engine is still worging at that time. 


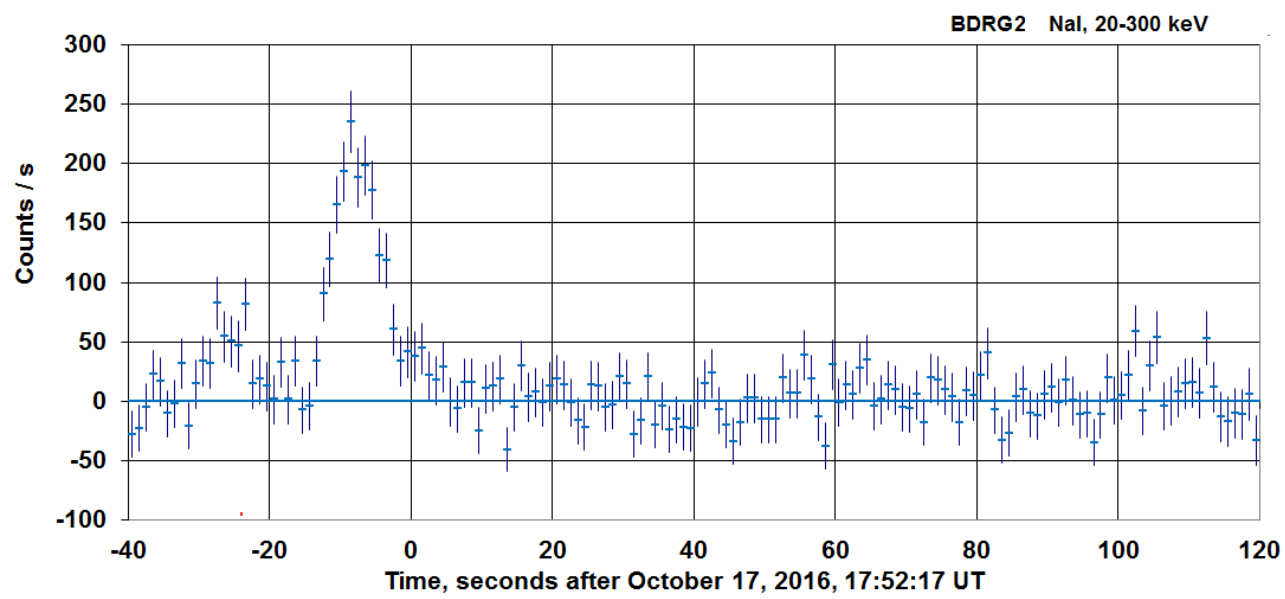

Fig. 3 Light curve of GRB171016A measured by BDRG2 onboard Lomonosov

GRB 161017A was also detected by coding mask telescope BAT onbord Swift mission [9] that determined the source position with enough accuracy. Then robotic telescopes of MASTER network developed in the M.V. Lomonosov Moscow State University were pointed to the burst source location and begun optical observations and ground robot MASTER located near Blagoveshchensk automatically recorded this event in the optical range (see fig4). One can see that optical transient appeared at $\sim 70 \mathrm{~s}$ from the Swift trigger that was produce at the front osf the first peak of the light curve i.e. about 30s before the BDRG/Lomonosov GRB trigger. So the optical emission corresponds to the period of the presence of prompt gamma-radiation. It is necessary to note that due to the almost robotized observation technique just MASTER telegram [11] was the first on the NASA site. This telegram informed about the discovery of GRB optical emission, which was sent by robot itself.
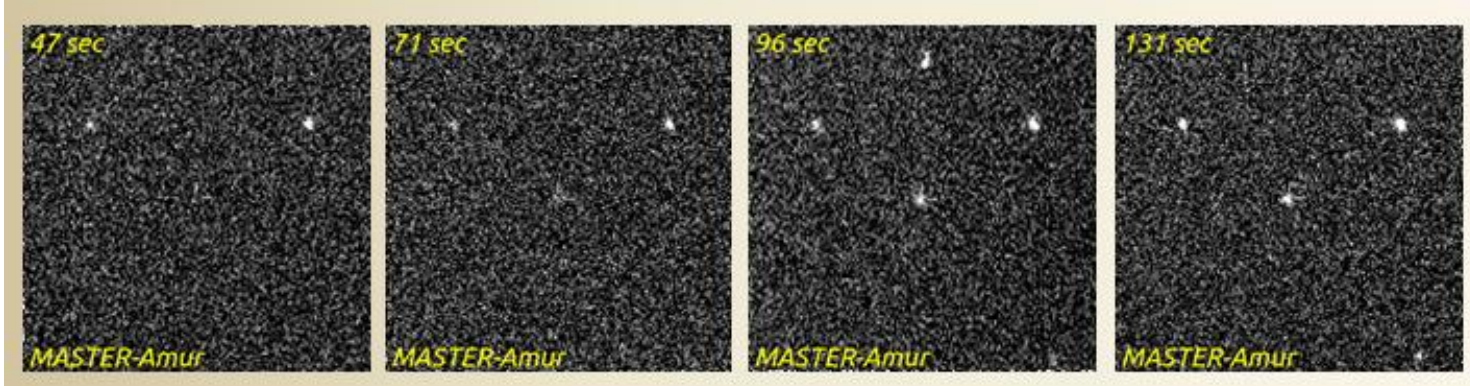

Fig.4. Images of optical transient of GRB 161017A obtained by MASTER-Amur robotic telescope

The combined light curve of GRB 161017 from Lomonosov, MASTER-net and Swift observations is presented at fig. 5. It is known from several previous observations that prompt optical emission detected during the first few minutes after the onset of a GRB in some cases correlates with the prompt $\gamma$ emission, and in other cases early optical light curve is uncorrelated with the $\gamma$-ray light curve [10]. One can see from fig.5 that in case of GRB 161017A optical emission is uncorrelated with Xray and Gamma. Xray and Gamma light curves have a similar structure, but optical emission rise up during more less quite time from 50 till $\sim 120$ s and even 
decay during second activity episode after $\sim 130$ s. Nevertheless, it should be noted that after 130 seconds in all three energy ranges there are two small peaks. It is interesting that, despite the similar structure, they are displaced relative to each other in time. A delay of longer wavelength radiation is observed with respect to the short-wavelength radiation.

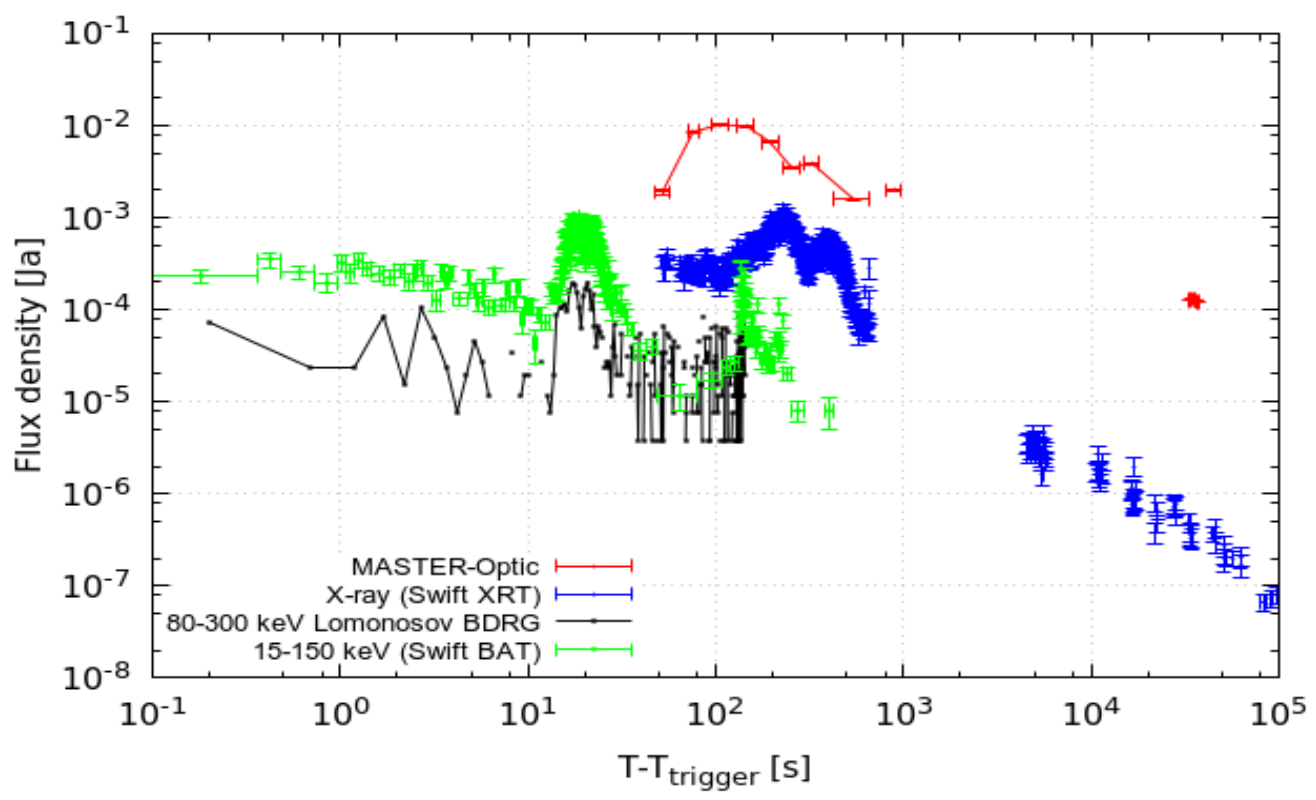

Fig. 5. Lomonosov, MASTER-net and Swift observations of GRB 161017A in broad bands from optics to gamma

\section{Conclusion}

The early optical observations of GRBs were realized in Lomonosov space mission by real time recording of the sequence of images from wide-FOV cameras. The joint observations of Lomonosov GRB monitor with MASTER net of robotic telescopes allowed to compare optical and light curve of the long-duration GRB 161017A at different phases of its activity.

\section{Acknowledgments}

The experiment onboard the Lomonosov satellite was realized with the partial financial support of M.V. Lomonosov Moscow State University within the framework of the "Prospects for Development" program ("Perspektivnye Napravleniya Razvitiya").

\section{References}

[1] A. M. Amelushkin, V. V. Bogomolov, V. V. Benghin, et.al. Space experiments on-board of Lomonosov mission to study gamma-ray bursts and UHECRs. EAS Publications Series, 61:545$552,2013$.

[2] V. A. Sadovnichiy, A. M. Amelyushkin, V. Angelopoulos, et al., Space experiments aboard the Lomonosov MSU satellite. Cosmic Research (English translation of Kosimicheskie Issledovaniya), 51:427-433, 2013.

[3] Gehrels, N. \& Razzaque, Gamma-ray bursts in the swift-Fermi era , S. Front. Phys. (2013) 8: 661. doi: $10.1007 / \mathrm{s} 11467-013-0282-3$ 
[4] A. M. Amelushkin, V. V. Bogomolov, V. I. Galkin, et.al. BDRG and SHOK instruments for study of GRB prompt emission in Michaylo Lomonosov space mission. EAS Publications Series, 61:553559,2013

[5] T. Sakamoto, A. Yoshida, Y. Kawakubo, GRB 160908A: CALET Gamma-Ray Burst Monitor detection GRB Coordinates Network, 19903:p.1, 2016

[6] GRB catalogue on the site of Lomonosov space mission, http://downloader.sinp.msu.ru/grb_catalog/

[7] A. M. Amelushkin, et.al.. GRB160720a: Lomonosov BDRG gamma ray detection and MASTER limit. GRB Coordinates Network, 19728: p.1, 2016.

[8] N. L. Dzhioeva, A. M. Amelushkin, V. O. Barinova, et al. GRB 160824b: Lomonosov BDRG gamma ray detection and MASTER limit. GRB Coordinates Network, 19884:p. 1, 2016.

[9] Troja, E.,et.al., GRB 161017A: Swift detection of a burst with a bright optical afterglow.',GRB Coordinates Network, Vol. 20064, p1, 2016

[10] E.S. Gorbovskoy, G.V. Lipunova, V.M. Lipunov, V.G. et al , Prompt, early and afterglow optical observations of five -ray bursts: GRB 100901A, GRB 100902A, GRB 100905A, GRB 100906A and GRB 101020A. MNRAS 421, 1874-1890 (2012). doi:10.1111/j.1365-2966.2012.20195.x

[11] V.Yurkov, Yu.Sergienko, D.Varda et al. GRB161017.74 MASTER-Net OT detection, GRB Coordinates Network, 20063:p. 1, 2016

\section{Lomonosov-GRB collaboration}

Mikhail Panasyuk, Sergey Svertilov, Vitaly Bogomolov, Alexander Amelushkin, Vera Barinova, Andrey Bogomolov, Anatoly lyudin, Natalia Dzhioeva, Vladimir Kalegaev, Polina Kazarjan, Ekaterina Kuznetsova, Alexander Lukin, Irina Myagkova, Alaxander Minaev, Anna Shustova, Min Nguyen, Vasily Petrov, Ivan Yashin

Lomonosov Moscow State University, Skobeltsyn Institute of Nuclear Physics, Physical department 1(2), Leninskie gory, GSP-1, Moscow, Russia

E-mail: bogoviterambler.ru

\section{Vladimir Lipunov, Evgeny Gorbovskoy, Viktor Kornilov}

Lomonosov Moscow State University, Sternberg Astronomical Institute Universitetsky pr., 13,Moscow, Russia

E-mail: lipunov2007@gmail.com

\section{H. Park, H.M. Jeong, Soomin Jeong, Minbin Kim}

Department of Physics, Sungkyunkwan University

Seobu-ro, Jangangu, Suwonsi, Korea

E-mail: ilparkeskku.edu

\section{Alberto Castro-Tirado}

Instituto de Astrofisica de Andalucia (IAA-CSIC)

P.O.Box 03004, E-18080, Granada, Spain

E-mail: ajcteiaa.es 